\title{
Estimação da velocidade média em vias arteriais urbanas com uso do microssimulador VISSIM
}

\author{
Victor Macêdo Lacerda ${ }^{1}$, Leonardo Gonçalves Ribeiro ${ }^{2}$, Matheus Ferreira da Rocha ${ }^{3}$, \\ Diego Alves Tabosa ${ }^{4}$, Manoel Mendonça de Castro Neto ${ }^{5}$
}

1Departamento de Engenharia de Transportes, Universidade Federal do Ceará, victor.macedo.ufc@gmail.com

2Departamento de Engenharia de Transportes, Universidade Federal do Ceará, leo.ribeiro@det.ufc.br

${ }^{3}$ Departamento de Engenharia de Transportes, Universidade Federal do Ceará, matheus.ferreira@det.ufc.br ${ }^{4}$ Departamento de Engenharia de Transportes, Universidade Federal do Ceará, diegomrsmim@ @otmail.com

${ }^{5}$ Departamento de Engenharia de Transportes, Universidade Federal do Ceará, manoel@det.ufc.br

\section{Recebido:}

29 de maio de 2018

Aceito para publicação:

5 de dezembro de 2018

Publicado:

31 de dezembro de 2019

Editor de área:

Sara Ferreira

\section{Palavras-chaves:}

Microssimulação de tráfego,

Car-following,

Mudança de faixa,

Wiedemann.

Keywords:

Traffic microsimulation,

Car-following,

Mudança de faixa,

Wiedemann.

DOI:10.14295/transportes.v27i4.1705
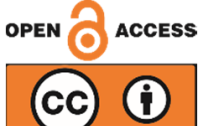

\begin{abstract}
RESUMO
Um dos principais desafios na modelagem microscópica de vias urbanas é a estimação dos valores dos parâmetros dos modelos comportamentais do condutor. $O$ principal objetivo deste artigo é propor um método de calibração dos modelos comportamentais do VISSIM para modelagem do tráfego de vias arteriais urbanas, com foco na estimação da velocidade média do tráfego de automóveis e de ônibus. O método foi aplicado em dois corredores urbanos da cidade de Fortaleza, resultando em erros de calibração de $10 \% \mathrm{e}$ $13 \%$ e de validação de $19 \%$ e $9 \%$. O segundo objetivo deste artigo foi comparar a calibração apresentada, do tipo sequencial, na qual os parâmetros são calibrados separadamente, seguindo uma sequência pré-estabelecida, com a calibração simultânea, na qual todos os parâmetros são calibrados em conjunto com base na medida de desempenho do tráfego que se deseja estimar. A calibração sequencial resultou em melhores estimativas para os parâmetros comportamentais, pois ela diminui a chance de se obter combinações de valores irreais para os parâmetros.
\end{abstract}

\begin{abstract}
One of the main challenges in urban traffic microscopic modeling is the estimation of the parameter of the driving behaviour models. The main objective of this paper is to propose a calibration methodology for VISSIM for modeling urban arterial roads, focusing on the estimation of the mean speed of cars and buses. The methodology was applied in two urban corridors in Fortaleza, resulting in calibration errors of $10 \%$ and $13 \%$ and validation errors of $19 \%$ and $9 \%$. The second objective of this paper was to compare the proposed sequential calibration with the simultaneous calibration, in which all parameters are calibrated together based on the traffic measure of effectiveness to be estimated. The sequential calibration resulted in better estimates of the parameters, as this methodology decreases the chances of obtaining unrealistic sets of parameter values.
\end{abstract}

\section{INTRODUÇÃO}

Os principais microssimuladores de tráfego veicular apresentam basicamente quatro modelos comportamentais: car-following, lane-changing, gap-acceptance e route choice. Um dos principais problemas na modelagem microscópica de vias urbanas é a estimação dos valores dos parâmetros de tais modelos, tarefa que vai desde a seleção dos parâmetros a serem estimados até a forma como eles serão calibrados. Hollander e Liu (2008) apresentam a discussão entre se realizar a calibração de forma sequencial, na qual os parâmetros são calibrados separadamente, 
seguindo uma sequência pré-estabelecida, e a calibração simultânea, em que todos os parâmetros são ajustados ao mesmo tempo, tendo como medida-alvo a própria medida de desempenho do tráfego que se deseja estimar. Este último tipo é o comumente encontrado na literatura (Karakikes, 2017; Liu et al., 2012; Lidbe et al., 2016; Park e Qi, 2005)

0 objetivo principal deste artigo é propor um método de calibração dos modelos comportamentais do VISSIM para a modelagem do tráfego de vias arteriais urbanas, tendo como foco a estimação da velocidade média dos automóveis e dos ônibus. Um segundo objetivo deste trabalho é comparar as estratégias de calibração sequencial e simultânea. 0 método proposto foi aplicado na modelagem de duas importantes vias arteriais da cidade de Fortaleza.

\section{MODELOS MICROSCÓPICOS DO VISSIM}

Como o objetivo deste trabalho é a modelagem da velocidade média na via arterial, foram considerados apenas os modelos comportamentais de car-following e de lane-changing. 0 modelo de car-following do VISSIM para vias urbanas, Wiedemann-74 (W74), possui três parâmetros. 0 primeiro é o $a x$, que representa o espaçamento entre os veículos quando parados em fila. 0 valor default do ax é 2,0 m com uma variação segundo uma distribuição normal com desvio padrão 0,3 m, truncada entre os valores 1,0 m e 3,0 m. A distância mínima de following é dada pela soma de $a x+b x$, sendo $b x$ função dos parâmetros bx_add (default 2,0 m), bx_mult (default 3,0 m) e da velocidade instantânea do veículo.

Pouco se tem dado atenção na literatura, mas além dos parâmetros de Wiedemannn, o VISSIM traz outros nove parâmetros no seu algoritmo de car-following. Neste artigo, esse conjunto de nove parâmetros será denominado parâmetros "não-Wiedemann". Os três primeiros parâmetros referem-se ao look ahead distance ( $L A D)$, os quais permitem o usuário modelar as distâncias mínima (MinLAD) e máxima (MaxLAD) que o condutor pode observar à frente, ou ao seu lado, e o número de veículos $(\mathrm{OV})$ observados à frente, para reagir a um determinado evento. 0 MinLAD só é utilizado se os veículos são autorizados a conduzir lado a lado em uma mesma faixa, como o caso de carros e bicicletas. Contudo, observou-se que este parâmetro pode influenciar medidas de desempenho como a velocidade média mesmo quando veículos não estão conduzindo lado a lado em uma mesma faixa. Para essa verificação, redes hipotéticas foram criadas e foram avaliadas as diferenças entre os tempos de viagem. Mesmo não havendo ultrapassagens em uma mesma faixa, o parâmetro MinLAD apresentou diferenças, embora insignificantes, na velocidade média. Isso ocorre porque o condutor levará em consideração inicialmente o número de veículos à sua frente $(O \mathrm{~V})$, seguido pelo MinLAD e o MaxLAD. Tomando como exemplo o valor default de 4 veículos a serem observados, o MinLAD só irá interferir no following caso o seu valor ultrapasse a posição do $4^{\underline{0}}$ veículo à frente. Essa medida, no meio urbano (espaçamento pequeno entre os veículos), com parâmetros default de W74, está em torno de 35m. Qualquer outro valor abaixo deste não terá influência, pois o que prevalecerá é a posição do 4ํo veículo à frente (parâmetro $O \mathrm{~V}$ ), e não mais o MinLAD.

Existem dois parâmetros relacionados ao look back distance (LBD) que definem a distância que um condutor pode observar à montante, de forma a reagir a um evento como mudança de faixa ou inserção em uma determinada via. Além desses parâmetros, outros dois podem ser ativados ou desativados pelo usuário e foram adicionados ao VISSIM somente nas versões mais recentes. 0 primeiro deles é o smooth closeup behavior, o qual determina o quanto suavemente o veículo desacelera ao se aproximar de um obstáculo (Aghabayk et al., 2013). Se habilitado, os veículos, ao perceberem algum obstáculo ou necessitarem de alguma reação, irão desacelerar 
uniformemente, iniciando a desaceleração desde o valor máximo do LAD (por default $250 \mathrm{~m}$ ) até o obstáculo. Esse comportamento gera uma desaceleração mais suave, pois se inicia a uma distância considerável. Se desabilitado (default), o veículo seguidor assume um comportamento normal de following, independente da presença de um obstáculo, até a velocidade do veículo líder cair para menos de $1 \mathrm{~m} / \mathrm{s}$ e venha a se tornar uma "impedância" para o seu trajeto.

O parâmetro standstill distance for static obstacles determina a distância que o veículo, quando parado, mantém dos obstáculos estáticos, como marcadores de sinais de 'Pare', regras de prioridade ou áreas de conflito, por exemplo. Se desabilitado (default), os veículos utilizam um valor aleatório que segue uma distribuição Normal, com média de 0,5 m e variância de $0,15 \mathrm{~m}^{2}$. Se habilitado, os veículos utilizam um valor determinado pelo usuário, que por default é $0,5 \mathrm{~m}$.

Por fim, dois parâmetros que merecem destaque nesta pesquisa são os relacionados ao temporary lack of attention (TLA), que representam a não reação momentânea do condutor ao estímulo do veículo à sua frente devido à falta de atenção. Os parâmetros procuram simular o efeito de variáveis como uso de telefones celulares e de equipamentos de som. No primeiro parâmetro, duration of temporary lack of attention (dur_TLA), o usuário define a duração da falta de atenção, que por default é zero segundos. No segundo parâmetro, probability of temporary lack of attention (prob_TLA), define-se a frequência com que esta ocorre, que por default é 0,0\%. 0 aumento desses dois parâmetros (duração e frequência) diminui a capacidade e a velocidade média na via, visto que estes parâmetros retardam a partida dos condutores.

No modelo de lane-changing do VISSIM, o veículo inicia a tentativa de mudança de faixa a partir do parâmetro lane change distance, com o limite para realizar esta mudança até a emergency stop distance do seu conector de destino. Ao longo desse intervalo, a agressividade da mudança de faixa é dada pelas taxas de desaceleração do veículo que deseja mudar de faixa e do veículo vizinho. Os demais parâmetros ditam o grau de facilidade das mudanças de faixa, interferindo na brecha aceitável (minimum headway front/rear) e na distância de segurança (safety distance reduction factor), na cooperação do veículo vizinho (cooperative braking e cooperative lane change), e nas facilidades de manobras (advanced merging e lateral correction of rear end position). Caso o veículo não consiga realizar a mudança nesse intervalo definido, ele irá parar no limite da emergency stop distance e irá aguardar o aparecimento de uma brecha, com o limite de espera definido pelo parâmetro waiting time before diffusion.

Ao observar os conceitos dos parâmetros de lane-changing, percebe-se que dois exercem influência no comportamento de following, consequentemente, na velocidade média. Esses dois parâmetros são o minimum headway front/rear (min_head_fr) e o safety distance reduction fator (safe_dist_rf). 0 primeiro é a distância mínima entre a frente do veículo e a traseira do veículo à frente, após a mudança de faixa. 0 segundo é uma redução da distância de segurança para permitir a realização da mudança de faixa. 0 parâmetro possui valor default 0,6 , o que reduz a distância de segurança em 40\%. Quanto menores forem os valores destes parâmetros, maior a facilidade da mudança de faixa. Mais detalhes sobre esses e outros parâmetros comportamentais do VISSIM podem ser encontrados em Lacerda (2016).

\section{MÉTODO PROPOSTO}

0 método proposto, apresentado na Figura 1, é composto por duas fases. A primeira fase é a de pré-calibração, cujo principal objetivo é selecionar os parâmetros que devem ser estimados 
diretamente em campo, os que devem ser calibrados por meio de tentativa-e-erro e os parâmetros cujos valores podem ser obtidos de estudos anteriores.

A primera etapa da fase de pré-calibração é a de compreensão dos parâmetros, que é feita por meio de análise da literatura e por experimentos com redes hipotéticas. Em seguida, após a etapa de Coleta de dados para codificação, na qual são coletados os principais dados de entrada do microssimulação, tem-se a etapa de análise do impacto dos parâmetros, na qual são realizados dois tipos de análise de sensibilidade para cada modelo. 0 primeiro consiste em variar um parâmetro por vez, mantendo-se os demais com valores default, tendo, portanto, o objetivo de se avaliar o efeito isolado do parâmetro nas medidas de desempenho. Cada parâmetro foi variado em dois níveis, mínimo e máximo, escolhidos a partir da literatura. Como esta abordagem traz a limitação de não considerar as dependências entre os parâmetros, realiza-se o segundo tipo de análise de sensibilidade, baseada na comparação de duas combinações de valores dos parâmetros: uma combinação que compõe o motorista de perfil "cauteloso" e outra combinação que representa o motorista "agressivo". O motorista cauteloso foi formado pelos valores dos limites de busca dos parâmetros que implicam em menores valores de velocidade média e de capacidade, ou seja, em maiores headways de following. 0 motorista agressivo foi formado pelos valores de busca que causam o efeito oposto.

É importante ressaltar que muitos trabalhos realizam testes estatísticos de igualdade de médias para avaliar a relevância de um parâmetro na medida de desempenho (Lownes e Machemehl, 2006). Há uma desvantagem nesta abordagem, que é o fato de que significância estatística não implica em significância prática. Em outras palavras, as médias podem ser estaticamente diferentes, porém a diferença entre elas pode não ser relevante para a aplicação em estudo. Por esta razão, neste trabalho são utilizados intervalos de confiança (IC) para avaliar o tamanho do efeito do parâmetro na velocidade média, e serão considerados os parâmetros que possuírem efeito significativo na medida de desempenho.

A fase de pré-calibração se encerra com a etapa de classificação dos parâmetros, na qual os parâmetros são agrupados de acordo com o proposto por Hollander e Liu (2008), ou seja, são agrupados os parâmetros que podem ser medidos diretamente em campo, os que precisam ser calibrados e os que podem ser encontrados em estudos similares.

A segunda fase, de calibração, tem como principal objetivo conceber e implementar uma estratégia de calibração que resulte em uma rede de simulação validada para os objetivos do estudo. Os parâmetros de calibração são divididos em blocos (resultado da etapa de definição dos blocos de parâmetros), sendo cada bloco definido como um conjunto de um ou mais parâmetros calibrados simultaneamente. Cada bloco possui a sua medida-alvo e o seu método de ajuste, que pode ser manual ou automatizado, com uso de algoritmos genéticos (AG), por exemplo, a depender do número de parâmetros do bloco. Deve-se formar blocos de tal forma que os parâmetros de um bloco sejam os únicos do modelo a exercerem impacto significativo na medida-alvo. Em seguida, parte-se para a coleta de dados para a calibração, em que serão coletadas as medidas-alvo definidas para cada bloco de parâmetros, seguida da configuração do $A G$, finalizando com a calibração e validação propriamente dita. Este tipo de calibração é chamado de sequencial porque os blocos de parâmetros são calibrados sequencialmente, embora os parâmetros dentro de um mesmo bloco sejam calibrados de forma simultânea. Por outro lado, na calibração denominada de simultânea todos os parâmetros são calibrados de uma só vez, ou seja, há somente um único bloco. 


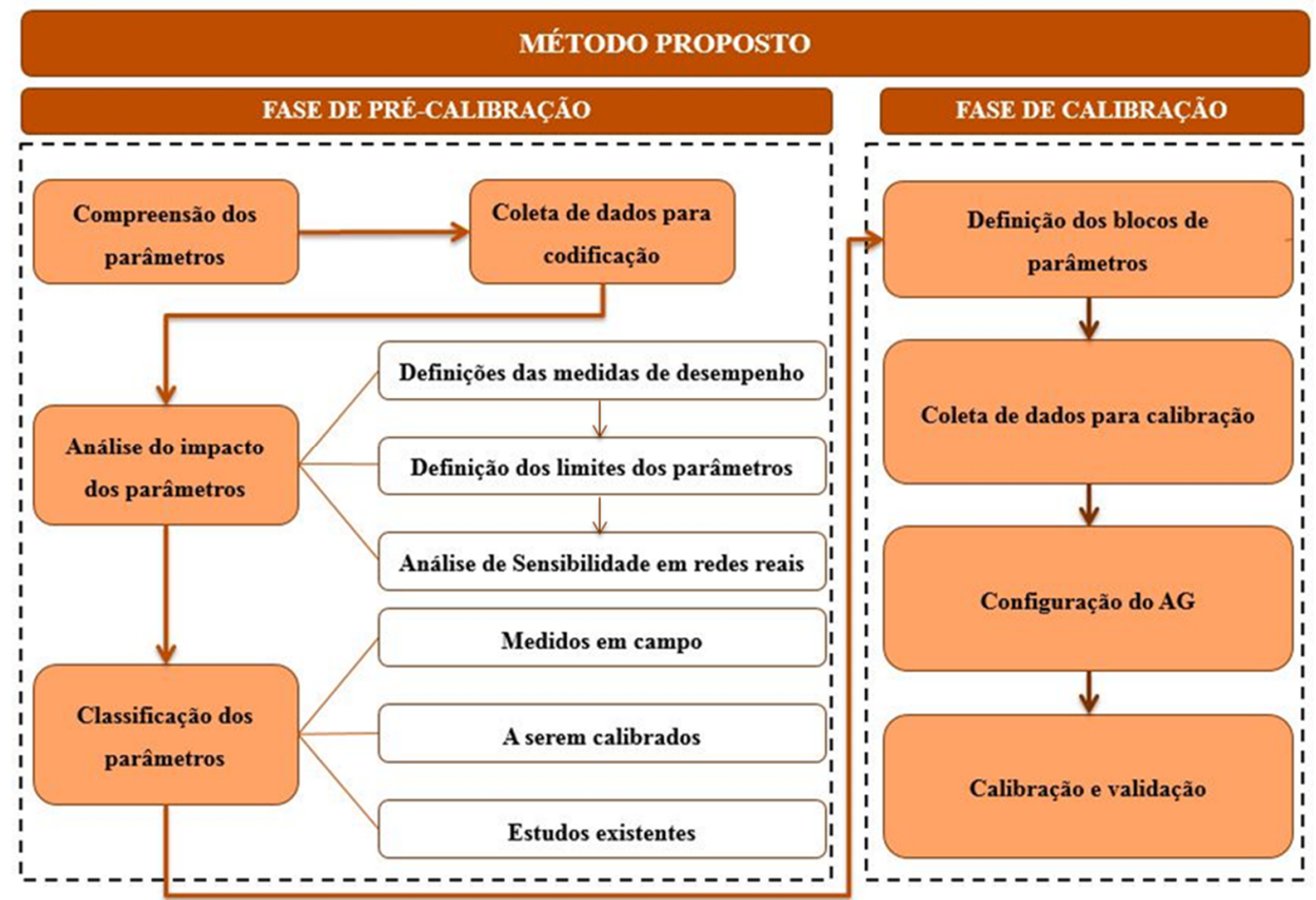

Figura 1. Método de calibração proposto

\section{4. ÁREAS DE ESTUDO}

Para a aplicação do método proposto nesta pesquisa, foram utilizados dois corredores arteriais de Fortaleza com características distintas, sendo o primeiro deles um trecho da Av. Santos Dumont, uma via arterial unidirecional com duas faixas de tráfego misto, localizada em uma das regiões mais adensadas da cidade. 0 trecho é composto por nove interseções em nível, das quais cinco são semaforizadas, totalizando cerca de $1,2 \mathrm{~km}$ de extensão. 0 trecho conta ainda com quatro pontos de parada de ônibus. Para fins de calibração e validação, o trecho escolhido da Av. Santos Dumont foi subdivido em dois subtrechos com aproximadamente a mesma extensão, para que um subtrecho fosse utilizado para calibração e outro para validação.

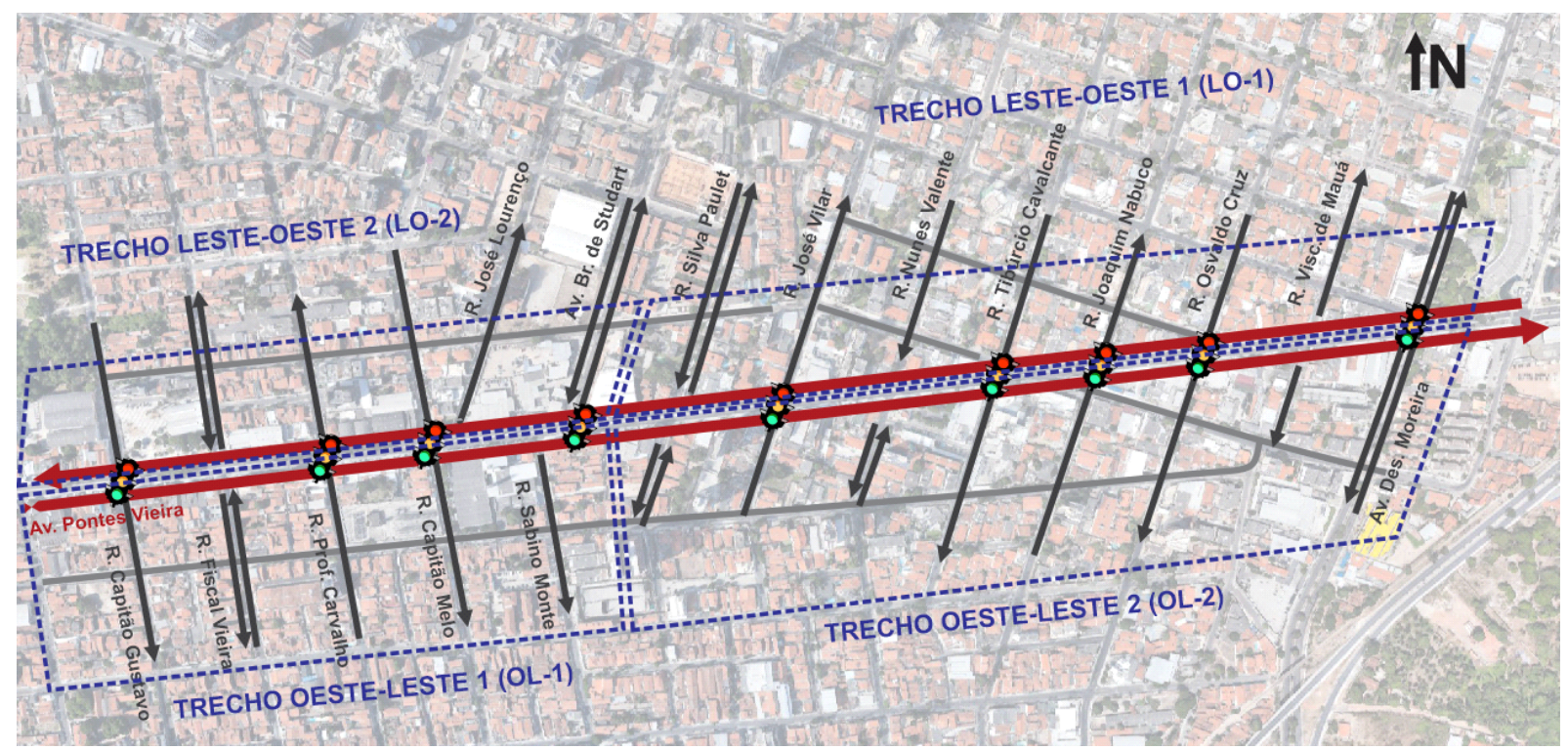

Figura 2. Corredor arterial Av. Pontes Vieira 
O segundo corredor é composto por um trecho da Av. Pontes Vieira, uma via arterial com 1,5 km de extensão e sentido duplo de circulação, com duas faixas de tráfego por sentido separadas por um canteiro central. A avenida é um importante corredor de ligação leste-oeste da cidade de Fortaleza. 0 trecho conta com 16 interseções em nível, sendo nove semaforizadas, e possui seis pontos de parada de ônibus por sentido. Por concisão, só é apresentada a figura da Av. Pontes Vieira (Figura 2).

\section{APLICAÇÃO}

Nesta seção é apresentada a aplicação do método proposto nos dois corredores. Serão discutidas as principais etapas das duas fases que compõem o método: pré-calibração e calibração.

\subsection{Pré-calibração}

A etapa de compreensão dos parâmetros se baseou na revisão da literatura e em experimentos realizados em redes hipotéticas. As principais conclusões desta etapa já foram apresentadas na Seção 2. A etapa de coleta de dados para a codificação incluiu dados relativos à demanda de automóveis, tempos semafóricos, headways e traçados das linhas de ônibus, tempo de serviço dos ônibus nas paradas e características físicas das vias. Portanto, somente automóves e ônibus foram incorporados na simulação. Os dados de demanda de automóveis direcional foram coletados através de contadores manuais, em todas as interseções dos corredores escolhidos, com períodos de agregação de 15 minutos. Para a Av. Santos Dumont, as coletas foram realizadas no período da manhã (de $6 \mathrm{~h} 00$ às $9 \mathrm{~h} 30$ ) de uma quarta-feira, dia 30 de abril de 2014, enquanto para a Av. Pontes Vieira, as coletas foram realizadas em duas quartas-feiras, dias 19 e 26 de outubro de 2016, no período de $7 \mathrm{~h}$ às $9 \mathrm{~h}$. Foram realizadas contagens classificatórias por auxílio de câmeras posicionadas em pontos específicos ao longo dos trechos definidos. Os dados relativos às programações dos semáforos foram obtidos junto ao Controle de Tráfego em Área de Fortaleza (CTAFOR). A Av. Santos Dumont conta com 10 linhas de transporte público numa demanda média de 42 ônibus/hora. Na Av. Pontes Vieira, a demanda na seção crítica foi de 78 ônibus/hora.

Em seguida, passou-se à análise do impacto dos parâmetros na velocidade média do tráfego de automóveis. As análises de sensibilidade foram feitas inicialmente para cada parâmetro isoladamente, mantendo os demais com valor default. Porém, a fim de se considerar a dependência entre os parâmetros, foram criados um perfil de motorista cauteloso e um agressivo para cada um dos modelos comportamentais analisados. A análise de sensibilidade avaliou todos os 45 parâmetros dos modelos de car-following e de lane-chaging, mas, por motivo de concisão, só serão mostrados os resultados dos 7 parâmetros comportamentais que se mostraram relevantes para a estimação da velocidade média nas vias. Como dito na seção 3, a relevância de cada parâmetro se baseou principalmente em IC para a diferença da velocidade média entre os níveis do parâmetro.

Na análise de sensibilidade dos parâmetros do modelo de car-following W74, foram definidos como limites os valores encontrados por Kim e Rillet (2005). Os limites de busca e os resultados encontram-se na Tabela 1. Para os parâmetros de car-following não-Wiedemann, dur_TLA e prob_TLA, os valores default já representam os valores baixos, iguais a zero. Como não há falta de atenção, considerou-se que os valores baixos desses parâmetros configuram condutores agressivos, por terem velocidades médias maiores, ao passo que os motoristas com valores altos foram considerados cautelosos. 0 valor alto foi definido de acordo com Warren (2016): dur_TLA 
igual a 1,0 s e prob_TLA igual a 10\%, como mostrado na Tabela 1. Os dois parâmetros foram variados simultaneamente pois possuem efeitos combinados, de modo que não há efeito ao se variar a duração do TLA se a frequência permanece em $0,0 \%$, por exemplo. Além disso, adotouse a premissa de que quanto mais distraído for o motorista, maior a frequência e a duração de suas distrações.

Com relação aos parâmetros de lane-changing, para o min_head_fr, quanto maior o seu valor, mais cauteloso é o condutor, sendo, portanto, o valor baixo atribuído a condutores agressivos. Park e Schneeberger (2003) utilizaram esse parâmetro com um valor máximo de 7,0 m para um estudo em rodovia. Porém, como essa distância é bastante alta para a realidade do tráfego urbano, adotou-se o valor de 3,0 m como limite superior neste trabalho. Para o parâmetro safe_dist_rf, quanto menor seu valor, maior será a redução da distância de segurança, portanto mais agressivo o condutor. Utilizou-se um valor alto de 0,8 e um valor baixo de 0,2 , como mostra a Tabela 1 .

Os três parâmetros do modelo de car-following W74 exerceram impacto bastante acentuado na velocidade média nas duas vias, com IC de 95\% para a diferença de 11,8 a 12,6 km/h na Av. Santos Dumont e de 10,1 a 11,2 km/h na Av. Pontes Vieira. Com relação aos parâmetros de carfollowing não-Wiedemann, o que mais exerceram efeito foram o dur_TLA e prob_TLA, embora IC da média das diferenças tenha englobado o valor zero. Para o modelo de lane-changing os parâmetros significativos foram os parâmetros min_head_fr e o safe_dist_rf. 0 IC (95\%) da diferença de velocidades entre o motorista agressivo e o cauteloso para a análise de sensibilidade dos parâmetos do modelo de lane-changing foi de 6,9 a 8,0 km/h na Av. Santos Dumont e de 12,8 a $13,9 \mathrm{~km} / \mathrm{h}$ na Av. Pontes Vieira, portanto bastante acentuado. Os detalhes das análises de sensibilidade podem ser encontrados em Lacerda (2016).

Além dos sete parâmetros dos modelos comportamentais, também foi considerado para a calibração o parâmetro velocidade desejada (vel_des), que é a velocidade do veículo quando estão desempedidos. Esse parâmetro pode exercer influência significativa na velocidade média para redes não congestionadas (Lacerda, 2014), e tem sido selecionado como parâmetro relevante em estudos de calibração do VISSIM (Khavas et al., 2017).

Os parâmetros foram classificados e agrupados em blocos. 0 primeiro bloco é composto pelos parâmetros a serem coletados em campo, devido à facilidade de coleta: ax e vel_des. No segundo bloco estão os parâmetros $b x_{-} a d d$ e $b x_{-}$mult, que serão calibrados manualmente e possuem a mesma medida-alvo, o fluxo de saturação, que está muito bem correlacionada com o conceito de ambos os parâmetros (Lacerda et al., 2014). No terceiro bloco estão os quatro parâmetros a serem calibrados simultaneamente com auxílio de um AG (dur_TLA, prob_TLA, min_head_fr, e safe_dist_rf) tendo como medida-alvo a medida de desempenho da via, ou seja, a velocidade média, já que não foram identificadas medidas de desempenho específicas para esses parâmetros neste trabalho. A razão para se utilizar um método de otimização para esse bloco é o número de parâmetros, quatro, que já torna benéfica a utilização de um método de busca automatizada. Os AGs têm sido um dos métodos mais utilizados na calibração de micsrossimuladores de tráfego (Buisson, et al., 2015). 
Tabela 1 - Análise de sensibilidade dos parâmetros comportamentais impactantes

\begin{tabular}{|c|c|c|c|c|c|c|}
\hline \multirow[b]{2}{*}{ Modelo } & \multicolumn{2}{|c|}{ Parâmetro } & \multicolumn{2}{|c|}{ Santos Dumont } & \multicolumn{2}{|c|}{ Pontes Vieira } \\
\hline & Default & Limites & $\begin{array}{c}\text { Vel. } \\
(\mathrm{Km} / \mathrm{h})\end{array}$ & $\begin{array}{c}\text { IC (95\%) } \\
(\mathrm{Km} / \mathrm{h})\end{array}$ & $\begin{array}{c}\text { Vel. } \\
(\mathrm{Km} / \mathrm{h})\end{array}$ & $\begin{array}{c}\text { IC (95\%) } \\
\text { (Km/h) }\end{array}$ \\
\hline \multirow{8}{*}{ W74 } & $A x$ & $1,0 \mathrm{~m}$ & 20,3 & \multirow{2}{*}{$-4,9 ;-3,2$} & 28,4 & \multirow{2}{*}{$-1,7 ;-0,8$} \\
\hline & $(2,0 \mathrm{~m})$ & $4,0 \mathrm{~m}$ & 15,8 & & 27,1 & \\
\hline & bx_add & $1,0 \mathrm{~m}$ & 20,3 & \multirow{2}{*}{$-9,3 ;-8,3$} & 28,6 & \multirow{2}{*}{$-7,9 ;-6,6$} \\
\hline & $(2,0 \mathrm{~m})$ & $10,0 \mathrm{~m}$ & 11,4 & & 21,1 & \\
\hline & $b x \_m u l t$ & 1,0 & 20,3 & \multirow{2}{*}{$-6,2 ;-4,4$} & 28,9 & \multirow{2}{*}{$-3,6 ;-2,3$} \\
\hline & $(3,0)$ & 10,0 & 14,4 & & 25,7 & \\
\hline & Cauteloso & --- & 10,7 & \multirow{2}{*}{11,$8 ; 12,6$} & 18,9 & \multirow{2}{*}{10,$1 ; 11,2$} \\
\hline & Agressivo & --- & 22,7 & & 29,3 & \\
\hline \multirow{6}{*}{ Não-Wiedemann } & dur_TLA & $0,0 \mathrm{~s}$ & 213 & \multirow{4}{*}{$-1,8 ; 0,3$} & 283 & \multirow{4}{*}{$-0,5 ; 0,3$} \\
\hline & $(0,0 \mathrm{~s})$ & $0,0 \%$ & & & & \\
\hline & prob_TLA & $1,0 \mathrm{~s}$ & 20 & & 282 & \\
\hline & $(0,0 \%)$ & $10,0 \%$ & 20,2 & & & \\
\hline & Cauteloso & --- & 20,3 & \multirow{2}{*}{0,$8 ; 1,5$} & 27,8 & \multirow{2}{*}{0,$5 ; 0,9$} \\
\hline & Agressivo & --- & 21,3 & & 28,4 & \\
\hline \multirow{6}{*}{ Lane-changing } & Min_head_fr & $0,5 \mathrm{~m}$ & 19,5 & \multirow{2}{*}{$-1,1 ;-0,1$} & 28,3 & \multirow{2}{*}{$-1,4 ;-0,7$} \\
\hline & $(0,5 \mathrm{~m})$ & $3,0 \mathrm{~m}$ & 18,8 & & 27,2 & \\
\hline & Safe_dist_rf & 0,2 & 19,9 & \multirow{2}{*}{$-0,8 ; 0,0$} & 29,0 & \multirow{2}{*}{$-1,6 ;-0,8$} \\
\hline & $(0,6)$ & 0,8 & 19,5 & & 27,8 & \\
\hline & Cauteloso & --- & 20,4 & \multirow{2}{*}{6,$9 ; 8,0$} & 24,8 & \multirow{2}{*}{12,$8 ; 13,9$} \\
\hline & Agressivo & --- & 21,7 & & 29,2 & \\
\hline
\end{tabular}

\subsection{Fase de calibração}

Para a fase de calibração foram coletados, para ambos os corredores em análise, dados das seguintes variáveis: distância ente veículos parados (para o parâmetro $a x$ ), fluxo de saturação (para os parâmetros $b x_{-} a d d$ e $b x_{-}$mult), velocidade desejada (parâmetro o vel_des) e tempo dos ônibus nas paradas. A distância média entre os veículos parados ( $a x$ ) foi coletada através de filmagens aéreas obtidas através de drone e com o auxílio de mini-cones para estabelecimento da escala na imagem. De acordo com as imagens foram obtidas 54 observações de distância de parada, o que resultou em uma média de 2,2 m e um desvio padrão de 0,8 m. 0 VISSIM apresenta como default o valor de 2,0 m (médio) para o $a x$, seguindo uma distribuição Normal com desvio padrão de 1,0 m que não pode ser alterado pelo usuário, truncada entre os valores 1,0 e 3,0 metros.

As velocidades foram obtidas através de veículos-teste e por pesquisas de placas de automóveis e de ônibus. Para a Av. Pontes Vieira, no primeiro dia de coleta, com relação aos dados obtidos a partir dos veículos-teste, para o sentido oeste-leste foram obtidos 34 observações de velocidade média durante as duas horas de coleta e 31 observações para o sentido oposto. Para os dados obtidos das pesquisas de placas, no sentido oeste-leste foram obtidas 273 observações e no sentido oposto, 229. A coleta da velocidade desejada se deu com uso de um pocket radar, com o qual foram obtidas as velocidades dos veículos desempedidos, ou seja, trafegando sem veículos à frente e sem efeito de controle semafórico. A amostra resultou numa média de 45 $\mathrm{km} / \mathrm{h}$ e desvio padrão de $9 \mathrm{~km} / \mathrm{h}$, abaixo da velocidade máxima permitida da via de $60 \mathrm{~km} / \mathrm{h}$. Como o VISSIM já apresenta distribuições default para a velocidade desejada, optou-se por utilizar as existentes no software, alocando a distribuição de $40 \mathrm{~km} / \mathrm{h}$ para os ônibus e caminhões - com limites entre 40 e $45 \mathrm{~km} / \mathrm{h}$ e de $50 \mathrm{~km} / \mathrm{h}$ para os veículos leves - com limites entre 45 e $58 \mathrm{~km} / \mathrm{h}$. Os mesmos métodos foram replicados para o segundo dia de pesquisa. Para ambos os 
corredores, o fluxo de saturação foi coletado em campo, sendo um local de coleta para a Av. Santos Dumont e quatro locais de coleta para Av. Pontes Vieira. Quanto ao transporte público, os dados de tempo de permanência do ônibus na parada foram obtidos por meio de filmagens.

Para esta pesquisa foi desenvolvido um AG codificado em linguagem Python versão 2.7 para realizar a calibração automatizada dos parâmetros, linguagem essa que se comunica com VISSIM por meio de interface COM, permitindo fácil manipulação dos objetos e funções do microssimulador. A configuração do AG teve as seguintes características: a medida de ajuste foi o erro percentual absoluto médio (EPAM) entre a velocidades médias simuladas e observadas; os limites de busca de cada parâmetro foram os utilizados nas análises de sensibilidade realizadas neste trabalho; os valores dos parâmetros de controle e dos operadores genéticos foram o tamanho da população: 10; número máximo de gerações: 20; replicações: 5; diversidade: 2; taxa de mutação: $20 \%$ e taxa de predação: $20 \%$, tendo como base as análises realizadas no trabalho de Medeiros et al. (2013).

Em seguida passou-se à etapa de calibração e validação. Para a Av. Santos Dumont, utilizouse um subtrecho para a calibração e outro para a validação, ambos com dados coletados no mesmo dia. Com relação à Av. Pontes Vieira, havia 2 dias de coleta para a rede completa. Para o primeiro dia de coleta, observou-se inconsistência nos dados de volume com altas discrepâncias entre os intervalos de 15 minutos no período de $8 \mathrm{~h}$ às $9 \mathrm{~h}$. No segundo dia de coleta, três dos nove semáforos apresentaram problema no período de $7 \mathrm{~h}$ às $8 \mathrm{~h}$, o que causou um comportamento atípico do tráfego na via. Dessa forma, optou-se por descartar estes dados e utilizar apenas os dados do período de $7 \mathrm{~h}$ às $8 \mathrm{~h}$ do primeiro dia de coleta para a calibração, e o período de $8 \mathrm{~h}$ às $9 \mathrm{~h}$ do segundo dia para a validação.

Para a rede da Santos Dumont, a calibração resultou num EPAM de 10,3\%, bastante reduzido quando comparado ao valor de 31,0\% para os parâmetros em valores default. Para a rede da Av. Pontes Vieira, a avaliação foi desagreada por subtrecho. Com a rede em parâmetros default, o EPAM geral foi de 14\%, sendo o EPAM de cada subtrecho (OL-1 e 2 e LO-1 e 2) com valores de $23 \%, 11 \%, 8 \%$ e $12 \%$, respectivamente. Para a rede calibrada, os respectivos erros nos subtrechos foram de 16\%,12\%,15\% e 11\%, resultando num EPAM geral de 12,6\%. Embora o erro geral tenha ficado similar, o erro no trecho OL-1 diminuiu significativamente, contudo, o erro do trecho L0-1 aumentou. Esse aumento do erro se deu pelo fato do trecho LO-1 ser o mais congestionado da rede, apresentando grande sensibilidade à variação dos parâmetros.

Na validação, o EPAM com os parâmetros em default foi de $26 \%$ para a rede da Santos Dumont. Depois da calibração, o erro reduziu para 18,6\%. Na Av. Pontes Vieira, o erro de validação com os parâmetros default foi de $16 \%$; com a rede calibrada, o erro reduziu para $9 \%$.

Visando obter uma maior capacidade de representação do modelo, realizou-se a avaliação da velocidade média dos ônibus para o trecho em análise. Para o sentido oeste-leste, os dados de velocidade dos ônibus não puderam ser utilizados porque entre os pontos de coleta de placas existiam duas rotas de ônibus, uma seguindo pela Av. Pontes Vieira e outra seguindo pela via paralela a ela, e na coleta dos dados não foram especificados os ônibus que percorriam pela Av. Pontes Vieira, dificultando a identificação dos tempos de viagem de interesse. Porém, para o sentido leste-oeste, foi possível a obtenção dos dados. De acordo com o valor calculado, a velocidade média dos ônibus no sentido LO foi de 15,3 km/h. Com a rede calibrada e validada, a velocidade média obtida do simulador para os ônibus foi de $15,5 \mathrm{~km} / \mathrm{h}$, apresentando um erro percentual de apenas $1,5 \%$. Esses resultados reforçam a validação dos modelos 
comportamentais, uma vez que a velocidade média dos coletivos é afetada pela corrente de tráfego de automóveis.

\section{COMPARAÇÃO COM CALIBRAÇÃO SIMULTÂNEA}

Boa parte dos estudos envolvendo a calibração de microssimuladores apresenta a calibração do tipo simultânea, na qual todos os parâmetros de interesse são ajustados simultaneamente, geralmente tendo como medida-alvo a medida de desempenho que se deseja simular. Na visão dos autores deste trabalho, esse tipo de calibração deve ser evitado, pois a otimização poderá resultar numa combinação de valores de parâmetros irrealista. Nesta seção é comparada a simulação proposta neste trabalho, do tipo sequencial, com a calibração simultânea, na qual os oito parâmetros relevantes para este trabalho são calibrados simultaneamente com o AG. A Tabela 2 apresenta os resultados.

Tabela 2 - Comparação da calibração sequencial e simultânea

\begin{tabular}{ccccccccccc}
\hline & vel_des & ax & bx_add & bx_mult & $\begin{array}{c}\text { dur. } \\
\text { LAT }\end{array}$ & $\begin{array}{c}\text { prob. } \\
\text { LAT }\end{array}$ & $\begin{array}{c}\text { min_ } \\
\text { head_fr }\end{array}$ & safe_dist_rf & EPAM \\
\hline \multicolumn{10}{c}{ Av. Santos Dumont } \\
\hline Sequencial & 50 & 2,2 & 5 & 5 & 0,7 & $2,7 \%$ & 0,5 & 0,2 & $10,3 \%$ \\
Simultânea & 40 & 2,5 & 3,3 & 4,6 & 0,6 & $5,3 \%$ & 1,8 & 0,5 & $10,9 \%$ \\
\hline \multicolumn{10}{c}{ Av. Pontes Vieira } \\
\hline Sequencial & 50 & 2,2 & 5 & 5 & 0,5 & $0,1 \%$ & 2,8 & 0,8 & $12,6 \%$ \\
Simultânea & 40 & 2,1 & 2,5 & 3,0 & 0,7 & $1,0 \%$ & 1,4 & 0,7 & $11,6 \%$ \\
\hline
\end{tabular}

Na rede da Av. Santos Dumont a calibração simultânea resultou em velocidade desejada menor do que a encontrada em campo. 0 parâmetro ax ficou um pouco maior $(0,3 \mathrm{~m}$ por veículo) e o bx_add e o bx_mult foram menores do que os encontrados na calibração sequencial. Como há grande confiança nos valores desses parâmetros provenientes da calibração sequencial, isso significa que a calibração simultânea resultou em fluxos de saturação e capacidade viária superestimadas. De fato, o fluxo de saturação obtido através da calibração simultânea foi de 1621 vph, sendo 10\% mais alto que o valor coletado em campo (1476 vph), ao passo que o fluxo de saturação obtido da calibração sequencial foi de $1540 \mathrm{vph}$, portanto, apenas $4 \%$ maior que o valor observado em campo (Tabela 3). Como a subestimação desses quatro parâmetros ( $a x$, $b x_{-} a d d, b x_{-}$mult e vel_des) superestima a velocidade média, a calibração simultânea procurou compensar este erro com o aumento dos demais parâmetros: dur_TLA, prob_TLA, min_head_fr e safe_dist_rf. Valores altos de dur_TLA e prob_TLA decorrem de motoristas mais desatentos, cujos efeitos no tráfego (diminuição de capacidade e de velocidade) são os mesmos do perfil cauteloso. Um valor elevado para min_head_fr implica em mais cautela para os motoristas, já que eles só aceitarão distâncias maiores de following. Da mesma forma, um valor alto de safe_dist_rf, no caso 0,8 , sugere que a distância mínima de following seja reduzida somente em $20 \%$ das manobras de mudança de faixa. Portanto, a compensação resultou em um motorista com perfil contraditório, pois metade dos parâmetros apresenta valores de motorista com perfil agressivo e a outra metade com perfil cauteloso. Essa compensação nos parâmetros até resultou em valores de EPAM similares aos da calibração proposta, mas gerou valores inadequados, ou contraditórios, dos parâmetros.

Vale analisar os resultados da calibração do bloco de parâmetros (dur_TLA, prob_TLA, min_head_fr e safe_dist_rf.) da calibração sequencial, pois os quatro parâmetros foram calibra- 
dos simultaneamente com o AG, portanto poderiam gerar valores inconsisentes. Não foram encontradas inconsistências no que tange às relações de dependência entre os parâmetros. Porém, em uma das vias (Av. Santos Dumont) os valores dos dois parâmetros de lane-changing foram menores do que os encontrados na segunda via (Av. Pontes Vieira). Fica a dúvida se o comportamento de mudança de faixa realmente se difere nas duas vias, ou se a diferença nos parâmetros é inadequada e se deu devido à busca do AG pelo menor erro.

Tabela 3 - Comparação dos fluxos de saturação na calibração sequencial e simultânea

\begin{tabular}{cc|cc|cc|cc}
\hline $\begin{array}{c}\text { Fluxos de saturação } \\
\text { (FS) }\end{array}$ & FS coletado & FS default & $\begin{array}{c}\text { EPAM } \\
(\%)\end{array}$ & FS sequencial & $\begin{array}{c}\text { EPAM } \\
(\%)\end{array}$ & FS simultâneo & $\begin{array}{c}\text { EPAM } \\
(\%)\end{array}$ \\
\hline Av. Santos Dumont & 1476 & 2052 & $39 \%$ & 1540 & $4 \%$ & 1621 & $10 \%$ \\
\hline PV OLsul & 1477 & 1735 & $17 \%$ & 1331 & $-10 \%$ & 1529 & $4 \%$ \\
PV OLnorte & 1470 & 2240 & $52 \%$ & 1660 & $13 \%$ & 1901 & $29 \%$ \\
PV LOsul & 1544 & 2051 & $33 \%$ & 1445 & $-6 \%$ & 1816 & $18 \%$ \\
PV LOnorte & 1317 & 2038 & $55 \%$ & 1317 & $0 \%$ & 1798 & $37 \%$ \\
\hline
\end{tabular}

Ao analisar os fluxos de saturação obtidos da Av. Pontes Vieira (Tabela 3), observa-se o mesmo comportamento da rede da Av. Santos Dumont, onde a calibração simultânea resultou em valores mais elevados de FS, portanto superestimando as capacidades para as quatro faixas de tráfego coletadas ( $N a$ Tabela 3, a nomenclatura "PV OLsul" significa Pontes Vieira - sentido Oeste-Leste - faixa sul, tendo as outras nomenclaturas o mesmo padrão). Ainda com relação à Av. Pontes Vieira, o erro da calibração simultânea foi menor que o obtido da calibração sequencial. Porém, os valores de vel_des, $a x, b x_{-} a d d$ e $b x_{-}$mult foram ainda menores, o que significa uma maior superestimação da capacidade e da velocidade média. Enquanto os OL-1 e LO-1 melhoraram seus resultados, os trechos OL-2 e LO-2 pioraram (Figura 3). Isso já era esperado pois, da mesma forma como se deu na calibração, os quatro trechos apresentavam diferentes demandas, fazendo com que determinados trechos e em determinados intervalos de tempo os resultados fossem mais sensíveis às variações dos parâmetros. Assim como ocorreu na Av. Santos Dumont, o EPAM da calibração simultânea na Av. Pointes Vieira foi relativamente baixo, mas apresentou valores de parâmetros inconsistentes.

\section{CONCLUSÕES}

Dentres os mais de 45 parâmetros comportamentais estudados, 7 foram relevantes para o estudo: os três parâmetros do modelo de car-following W74 ( $a x, b x_{-} a d d$ e bx_mult); dois parâmetros de car-following não-Wiedemann (dur_ TLA e prob_TLA) e dois parâmetros de lane-changing ( $m i n_{-}$head_fr e safe_dist_rf). Além desses, também foi significativo o impacto do parâmetro vel_des.

Um desafio na seleção de parâmetros de calibração reside nas relações de interdependência entre eles (Kim e Mahmassani, 2011). Como uma forma de se considerar as interdependências na análise de sensibilidade realizadas neste trabalho, foram criados dois perfis de motorista a partir de duas combinações de parâmetros: um agressivo e um cauteloso. Essa diferenciação permitiu avaliar o efeito do modelo comportamental como um todo nas medidas de desempenho.

A calibração proposta foi sequencial em três blocos de parâmetros. 0 primeiro bloco, de parâmetros medidos diretamente em campo, conteve o vel_des e o $a x$, devido à facilidade de 
obtenção em campo. Em seguida passou-se a calibração do segundo bloco, formado pelos parâmetros $b x_{-} a d d$ e $b x_{-} m u l t$, tendo como medida-alvo o fluxo de saturação. Posteriormente calibrou-se o último bloco, formado pelos parâmetros dur_TLA, prob_TLA, min_head_fr e safe_dist_rf, utilizando um AG, tendo como medida-alvo a velocidade média da corrente.

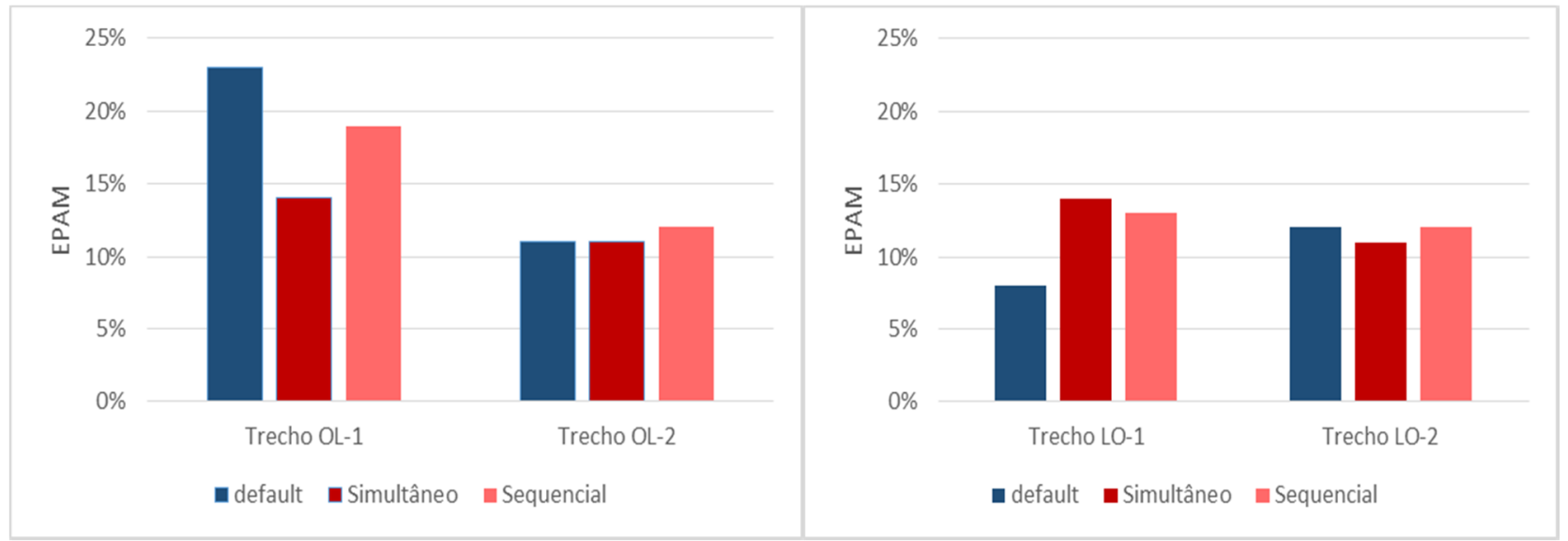

Figura 3. EPAM por subtrecho e estratégia de calibração - Av. Pontes Vieira

Como esperado pelos autores, a calibração sequencial se mostrou superior à calibração simultânea, pois embora os erros de estimativa da velocidade tenham sido similares, os valores dos parâmetros calibrados simultâneamente não foram bons. Esta conclusão também foi obtida no trabalho de Vasconcelos et al., (2014), que comparou a calibração sequencial com a simultânea para calibrar o modelo de car-following de Gipps.

A calibração feita em blocos bem definidos permite uma melhor estimativa dos parâmetros, pois evita-se o efeito de confundimento causado pela interferência dos outros parâmetros. 0 melhor tipo de bloco é o primeiro calibrado neste trabalho, em que os parâmetros são diretamente observados em campo, portanto é o tipo de bloco menos susceptível a erros. 0 segundo melhor tipo de bloco é o segundo calibrado neste artigo, em que os parâmetros (bx_add e bx_mult) não são observáveis diretamente em campo, mas eles são basicamente os únicos que afetam a sua medida-alvo (fluxo de saturação). Assim, isola-se o efeito de outros parâmetros nas estimativas. Por último, o tipo de bloco menos desejável -porém o mais comum na literatura- é o terceiro bloco calibrado neste trabalho, em que os parâmetros são ajustados tendo como medida-alvo a medida de desempenho de tráfego que se deseja estimar, no caso deste artigo, velocidade média, que é afetada por outros parâmetros do simulador. Por isto, além de bem escolhidos, é importante que os blocos sejam calibrados segundo a ordem cronológica aqui apresentada, pois sempre devem ser estimados primeiros os parâmetros menos passíveis de erros.

Sugere-se para trabalhos futuros a calibração dos parâmetros do terceiro bloco (dur_TLA, prob_TLA, min_head_fr e safe_dist_rf) em blocos do segundo tipo, ou seja, com medidas-alvo mais direcionadas aos parâmetros. Por exemplo, o parâmetro dur_TLA e prob_TLA podem ser calibrados usando uma medida-alvo baseada nos intervalos entre partidas dos veículos em aproximações semaforizadas, já que a falta de atenção dos motoristas tenderia a aumentar o valor dessa medida. Até o momento da submissão deste artigo, não foi encontrada na literatura nenhuma medida-alvo voltada especificamente para a calibração desses dois parâmetros. 
Sugere-se que estudos futuros testem a hipótese de que, quanto maior for o número de parâmetros a serem calibrados, maior será a necessidade de se adotar a calibração sequencial, pois maiores serão as chances de a calibração simultânea resultar em uma combinação inadequada de valores dos parâmetros.

Por fim, recomenda-se que estudos futuros incorporem modos de transportes que não foram modelados neste trabalho. A incorporação de motocicletas, pedestres e ciclistas deve tornar ainda mais clara a necessidade de se valer da calibração do tipo sequencial, já que a quantidade e a variedade de parâmetros no modelo de simulação aumentam consideravelmente.

\section{AGRADECIMENTOS}

O presente trabalho foi realizado com o apoio financeiro do Conselho Nacional de Desenvolvimento Científico e Tecnológico CNPq. Os autores também agradecem ao CTAFOR e à ETUFOR pela disponibilização de dados e de pessoal para coleta.

\section{REFERÊNCIAS}

Aghabayk, K., M. Sarvi, W. Young e L. Kautzsch (2013) A novel methodology for evolutionary calibration of vissim by multithreading. Australasian Transport Research Forum 2013 Proceedings. Brisbane, Australia.

Buisson, C., W. Daamen, V. Punzo, P. Wagner, M. Montanino e B. Ciuffo (2015) Calibration and validation principles. In: Daamen, W., C. Buisson e S. Hoogendorn (eds). Traffic Simulation and Data. Validation Methods and Applications. CRC Press. Florida, EUA.

Hollander, Y. e R. Liu (2008) The Principles of Calibrating Traffic Microsimulation Models. Transportation, v. 35, p. 347-362. DOI: $10.1007 / \mathrm{s} 11116-007-9156-2$.

Khavas, R., B. Hellinga e A. Masouleh (2017), Identifying Parameters to Model Traffic during Inclement Weather Using Microsimulation. Transportation Research Record. No. 2613, pp. 52-60. DOI: 10.3141/2613-07.

Karakikes, I, M. Spangler e M Margreiter (2017) Designing a Vissim-Model for a motorway network with systematic calibration on the basis of travel time measurements. Transportation Research Procedia, vol 24, p. 171-179. DOI: 10.1016/j.trpro.2017.05.086.

Kim, J. e H. S. Mahmassami (2011) Correlated Parameters in Driving Behavior Models. Transportation Research Record, $\mathrm{n}$. 2249, p 62-77. DOI: 10.3141/2249-09.

Kim, S-J, W. Kim, L.R. Rillet (2005) Calibration of Microsimulation Models Using Nonparametric Statistical Techniques. Transportation Research Record, n. 1935, p.111-119. DOI: 10.3141/1935-13.

Lacerda, V. e M. Castro-Neto (2014) Considerações sobre a calibração do modelo de car-following do vissim para vias arteriais urbanas. Anais do XXVIII Congresso da Associação Nacional de Pesquisa e Ensino em Transportes, ANPET, Curitiba, PR.

Lacerda, V. M. (2016) Estimação da Velocidade Média em vias urbanas com uso do microssimulador VISSIM. Dissertação de Mestrado, Programa de Mestrado em Engenharia de Transportes, Universidade Federal do Ceará, Fortaleza, CE.

Lidbe A. D., A. M. Hainen e S. L. Jones Jr. (2016) Comparative Study of Simulated Annealing and Genetic Algorithm for Calibration of Microsimulation Model. Transportation Research Board 95th Annual Meeting. Washington, USA.

Liu, P., X. Qu, H. Yu, W. Wang e B. Cao (2012) Development of a VISSIM Simulation Model for U-Turns at Unsignalized Intersections. Journal of Transportation Engineering, v. 138, n.11, p. 1333-1339. DOI:10.1061/(ASCE)TE.1943-5436.0000438.

Lownes, N. e R. Machemehl (2006) Sensitivity of Simulation Capacity to Modification of VISSIM Driver Behavior Parameters. Transportation Research Record, n. 1988, p. 102-110. DOI: 10.3141/1988-15.

Medeiros, A. L., M. M. Castro-Neto, C. F. Loureiro e J. E. Bessa-Jr (2013) Calibração de Redes Viárias Urbanas Microssimuladas com o uso de Algoritmos Genéticos. Anais do XXVII Congresso da Associação Nacional de Pesquisa e Ensino em Transportes, ANPET, Belém, PA.

Park, B. e J. D. Schneeberger (2003) Microscopic Simulation model calibration and Validation. Case Study of VISSIM Simulation Model for a Coordinated Actuated Signal System. Transportation Research Record, n. 1856, p. 185-192. DOI: 10.3141/1856-20.

Park, B e H. M. Qi. (2005) Development and Evaluation of a Procedure for the Calibration of Simulation Models. Transportation Research Record, no. 1934, p. 208-217. DOI: 10.3141/1934-22.

Vasconcelos, L., Neto, L. Santos, S., Silva, A.B. e Seco, A. Calibration of the Gipps Car-Following Model Using Trajectory Data. Transportation Research Procedia 3, pp. 952-961. DOI: 10.1016/j.trpro.2014.10.075.

Warren, A. (2016), Calibration of microsimulation models to field-measured saturation flow rates on signalized arterials. Transportation Research Board 95th Annual Meeting. Washington, USA. 